\title{
Creating Multilingual Translation Lexicons with Regional Variations Using Web Corpora
}

\author{
Pu-Jen Cheng, ${ }^{*}$ Yi-Cheng Pan*, Wen-Hsiang Lu ${ }^{+}$, and Lee-Feng Chien ${ }^{*}$ \\ ${ }^{*}$ Institute of Information Science, Academia Sinica, Taiwan \\ ${ }^{+}$Dept. of Computer Science and Information Engineering, National Cheng Kung Univ., Taiwan \\ Dept. of Information Management, National Taiwan University, Taiwan \\ \{pjcheng, thomas02, whlu, lfchien\}@iis.sinica.edu.tw
}

\begin{abstract}
The purpose of this paper is to automatically create multilingual translation lexicons with regional variations. We propose a transitive translation approach to determine translation variations across languages that have insufficient corpora for translation via the mining of bilingual search-result pages and clues of geographic information obtained from Web search engines. The experimental results have shown the feasibility of the proposed approach in efficiently generating translation equivalents of various terms not covered by general translation dictionaries. It also revealed that the created translation lexicons can reflect different cultural aspects across regions such as Taiwan, Hong Kong and mainland China.
\end{abstract}

\section{Introduction}

Compilation of translation lexicons is a crucial process for machine translation (MT) (Brown et al., 1990) and cross-language information retrieval (CLIR) systems (Nie et al., 1999). A lot of effort has been spent on constructing translation lexicons from domain-specific corpora in an automatic way (Melamed, 2000; Smadja et al., 1996; Kupiec, 1993). However, such methods encounter two fundamental problems: translation of regional variations and the lack of up-to-date and high-lexical-coverage corpus source, which are worthy of further investigation.

The first problem is resulted from the fact that the translations of a term may have variations in different dialectal regions. Translation lexicons constructed with conventional methods may not adapt to regional usages. For example, a Chinese-English lexicon constructed using a Hong Kong corpus cannot be directly adapted to the use in mainland China and Taiwan. An obvious example is that the word “taxi" is normally translated into “的士” (Chinese transliteration of taxi) in Hong Kong, which is completely different from the translated Chinese words of “出租车” (rental cars) in mainland China and “計 程車” (cars with meters) in Taiwan. Besides, transliterations of a term are often pronounced differently across regions. For example, the company name “Sony" is transliterated into “新力" (xinli) in Taiwan and “索尼” (suoni) in mainland China. Such terms, in today's increasingly internationalized world, are appearing more and more often. It is believed that their translations should reflect the cultural aspects across different dialectal regions. Translations without consideration of the regional usages will lead to many serious misunderstandings, especially if the context to the original terms is not available.

Halpern (2000) discussed the importance of translating simplified and traditional Chinese lexemes that are semantically, not orthographically, equivalent in various regions. However, previous work on constructing translation lexicons for use in different regions was limited. That might be resulted from the other problem that most of the conventional approaches are based heavily on domain-specific corpora. Such corpora may be insufficient, or unavailable, for certain domains.

The Web is becoming the largest data repository in the world. A number of studies have been reported on experiments in the use of the Web to complement insufficient corpora. Most of them (Kilgarriff et al., 2003) tried to automatically collect parallel texts of different language versions (e.g. English and Chinese), instead of different regional versions (e.g. Chinese in Hong Kong and Taiwan), from the Web. These methods are feasible but only certain pairs of languages and subject domains can extract sufficient parallel texts as corpora. Different from the previous work, Lu et al. (2002) utilized Web anchor texts as a comparable bilingual corpus source to extract translations for out-of-vocabulary terms (OOV), the terms not covered by general translation dictionaries. This approach is applicable to the compilation of translation lexicons in diverse domains but requires powerful crawlers and high network bandwidth to gather Web data.

It is fortunate that the Web contains rich pages in a mixture of two or more languages for some lan- 
guage pairs such as Asian languages and English. Many of them contain bilingual translations of terms, including OOV terms, e.g. companies', personal and technical names. In addition, geographic information about Web pages also provides useful clues to the regions where translations appear. We are, therefore, interested in realizing whether these nice characteristics make it possible to automatically construct multilingual translation lexicons with regional variations. Real search engines, such as Google (http://www.google.com) and AltaVista (http://www. altavista.com), allow us to search English terms only for pages in a certain language, e.g. Chinese or Japanese. This motivates us to investigate how to construct translation lexicons from bilingual searchresult pages (as the corpus), which are normally returned in a long ordered list of snippets of summaries (including titles and page descriptions) to help users locate interesting pages.

The purpose of this paper is trying to propose a systematic approach to create multilingual translation lexicons with regional variations through mining of bilingual search-result pages. The bilingual pages retrieved by a term in one language are adopted as the corpus for extracting its translations in another language. Three major problems are found and have to be dealt with, including: (1) extracting translations for unknown terms - how to extract translations with correct lexical boundaries from noisy bilingual search-result pages, and how to estimate term similarity for determining correct translations from the extracted candidates; (2) finding translations with regional variations - how to find regional translation variations that seldom cooccur in the same Web pages, and how to identify the corresponding languages of the retrieved searchresult pages once if the location clues (e.g. URLs) in them might not imply the language they are written in; and (3) translation with limited corpora - how to translate terms with insufficient search-result pages for particular pairs of languages such as Chinese and Japanese, and simplified Chinese and traditional Chinese.

The goal of this paper is to deal with the three problems. Given a term in one language, all possible translations will be extracted from the obtained bilingual search-result pages based on their similarity to the term. For those language pairs with unavailable corpora, a transitive translation model is proposed, by which the source term is translated into the target language through an intermediate language. The transitive translation model is further enhanced by a competitive linking algorithm. The algorithm can effectively alleviate the problem of error propagation in the process of translation, where translation errors may occur due to incorrect identification of the ambiguous terms in the intermediate language. In addi- tion, because the search-result pages might contain snippets that do not be really written in the target language, a filtering process is further performed to eliminate the translation variations not of interest.

Several experiments have been conducted to examine the performance of the proposed approach. The experimental results have shown that the approach can generate effective translation equivalents of various terms - especially for OOV terms such as proper nouns and technical names, which can be used to enrich general translation dictionaries. The results also revealed that the created translation lexicons can reflect different cultural aspects across regions such as Taiwan, Hong Kong and mainland China.

In the rest of this paper, we review related work in translation extraction in Section 2. We present the transitive model and describe the direct translation process in Sections 3 and 4, respectively. The conducted experiments and their results are described in Section 5. Finally, in Section 6, some concluding remarks are given.

\section{Related Work}

In this section, we review some research in generating translation equivalents for automatic construction of translational lexicons.

Transitive translation: Several transitive translation techniques have been developed to deal with the unreliable direct translation problem. Borin (2000) used various sources to improve the alignment of word translation and proposed the pivot alignment, which combined direct translation and indirect translation via a third language. Gollins et al. (2001) proposed a feasible method that translated terms in parallel across multiple intermediate languages to eliminate errors. In addition, Simard (2000) exploited the transitive properties of translations to improve the quality of multilingual text alignment.

Corpus-based translation: To automatically construct translation lexicons, conventional research in MT has generally used statistical techniques to extract translations from domain-specific sentencealigned parallel bilingual corpora. Kupiec (1993) attempted to find noun phrase correspondences in parallel corpora using part-of-speech tagging and noun phrase recognition methods. Smadja et al. (1996) proposed a statistical association measure of the Dice coefficient to deal with the problem of collocation translation. Melamed (2000) proposed statistical translation models to improve the techniques of word alignment by taking advantage of preexisting knowledge, which was more effective than a knowledge-free model. Although high accuracy of translation extraction can be easily achieved by these techniques, sufficiently large parallel corpora for 


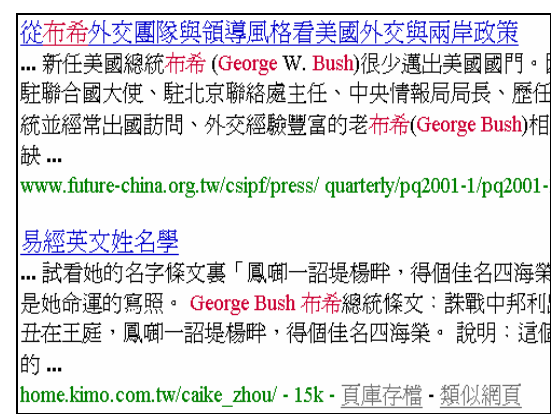

G8峰會人物志：美國總統布代

... G8峰會人物志：美國總統布什. 喬治? 沃克? 布什 (George V 年 7 月 6 日出生, 在得克薩斯州的米德闌和休斯敦長大, 其 屆總統喬治?布什。小布什畢業於耶鲁大學並獲學士學位 www.people.com.cn/BIG5/guoji 25/96/20030530/1004456.html

人物簡介：美國總統喬治. 布什

... 喬治. 沃克 . 布什 (George Walker Bush)栵稱小布什, 日出生, 在得克薩斯州的米德闌和休斯敦長大, 其父鳥 届總統喬治。布什。小布什畢業于耶魯大學并獲學士盆 www.people.com.cn/BIG5/shizheng/252/7429/7439/200202 [ www.people.com.cn 的其它相關資訊]

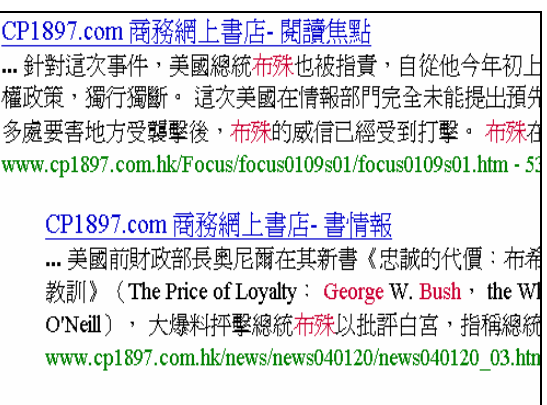

(a) Taiwan (Traditional Chinese) (b) Mainland China (Simplified Chinese) (c) Hong Kong (Traditional Chinese)

Figure 1: Examples of the search-result pages in different Chinese regions that were obtained via the English query term "George Bush" from Google.

various subject domains and language pairs are not always available.

Some attention has been devoted to automatic extraction of term translations from comparable or even unrelated texts. Such methods encounter more difficulties due to the lack of parallel correlations aligned between documents or sentence pairs. Rapp (1999) utilized non-parallel corpora based on the assumption that the contexts of a term should be similar to the contexts of its translation in any language pairs. Fung et al. (1998) also proposed a similar approach that used a vector-space model and took a bilingual lexicon (called seed words) as a feature set to estimate the similarity between a word and its translation candidates.

Web-based translation: Collecting parallel texts of different language versions from the Web has recently received much attention (Kilgarriff et al., 2003). Nie et al. (1999) tried to automatically discover parallel Web documents. They assumed a Web page's parents might contain the links to different versions of it and Web pages with the same content might have similar structures and lengths. Resnik (1999) addressed the issue of language identification for finding Web pages in the languages of interest. Yang et al. (2003) presented an alignment method to identify one-to-one Chinese and English title pairs based on dynamic programming. These methods often require powerful crawlers to gather sufficient Web data, as well as more network bandwidth and storage. On the other hand, Cao et al. (2002) used the Web to examine if the arbitrary combination of translations of a noun phrase was statistically important.

\section{Construction of Translation Lexicons}

To construct translation lexicons with regional variations, we propose a transitive translation model $S_{\text {trans }}(s, t)$ to estimate the degree of possibility of the translation of a term $s$ in one (source) language $l_{s}$ into a term $t$ in another (target) language $l_{t}$. Given the term $s$ in $l_{s}$, we first extract a set of terms $C=\left\{t_{j}\right\}$, where $t_{j}$ in $l_{t}$ acts as a translation candidate of $s$, from a corpus. In this case, the corpus consists of a set of search-result pages retrieved from search engines using term $s$ as a query. Based on our previous work (Cheng et al., 2004), we can efficiently extract term $t_{j}$ by calculating the association measurement of every character or word n-gram in the corpus and applying the local maxima algorithm. The association measurement is determined by the degree of cohesion holding the words together within a word ngram, and enhanced by examining if a word n-gram has complete lexical boundaries. Next, we rank the extracted candidates $C$ as a list $T$ in a decreasing order by the model $S_{\text {trans }}(s, t)$ as the result.

\subsection{Bilingual Search-Result Pages}

The Web contains rich texts in a mixture of multiple languages and in different regions. For example, Chinese pages on the Web may be written in traditional or simplified Chinese as a principle language and in English as an auxiliary language. According to our observations, translated terms frequently occur together with a term in mixed-language texts. For example, Figure 1 illustrates the search-result pages of the English term "George Bush," which was submitted to Google for searching Chinese pages in different regions. In Figure 1 (a) it contains the translations “喬治布希” (George Bush) and “布 希" (Bush) obtained from the pages in Taiwan. In Figures 1 (b) and (c) the term "George Bush" is translated into “布什”(busir) or “布甚”(buson) in mainland China and “布殊”(busu) in Hong Kong. This characteristic of bilingual search-result pages is also useful for other language pairs such as other Asian languages mixed with English.

For each term to be translated in one (source) language, we first submit it to a search engine for locating the bilingual Web documents containing the term and written in another (target) language from a specified region. The returned search-result pages containing snippets (illustrated in Figure 1), instead of the documents themselves, are collected as a corpus from which translation candidates are extracted and correct translations are then selected.

Compared with parallel corpora and anchor texts, bilingual search-result pages are easier to collect and can promptly reflect the dynamic content of the Web. 
In addition, geographic information about Web pages such as URLs also provides useful clues to the regions where translations appear.

\subsection{The Transitive Translation Model}

Transitive translation is particularly necessary for the translation of terms with regional variations because the variations seldom co-occur in the same bilingual pages. To estimate the possibility of being the translation $t \in T$ of term $s$, the transitive translation model first performs so-called direct translation, which attempts to learn translational equivalents directly from the corpus. The direct translation method is simple, but strongly affected by the quality of the adopted corpus. (Detailed description of the direct translation method will be given in Section 4.)

If the term $s$ and its translation $t$ appear infrequently, the statistical information obtained from the corpus might not be reliable. For example, a term in simplified Chinese, e.g. 互联网 (Internet) does not usually co-occur together with its variation in traditional Chinese, e.g. 網際網路 (Internet). To deal with this problem, our idea is that the term $s$ can be first translated into an intermediate translation $m$, which might co-occur with $s$, via a third (or intermediate) language $l_{m}$. The correct translation $t$ can then be extracted if it can be found as a translation of $m$. The transitive translation model, therefore, combines the processes of both direct translation and indirect translation, and is defined as:

$S_{\text {trans }}(s, t)=$

$\left\{\begin{array}{lr}S_{\text {direcd }}(s, t), & \text { if } S_{\text {direcec }}(s, t)>\theta \\ S_{\text {indirect }}(s, t)=\sum_{\forall m} S_{\text {direct }}(s, m) \times S_{\text {direct }}(m, t) \times \varpi(m), \text { otherwise }\end{array}\right.$

where $m$ is one of the top $k$ most probable intermediate translations of $s$ in language $l_{m}$, and $\varpi$ is the confidence value of $m$ 's accuracy, which can be estimated based on $m$ 's probability of occurring in the corpus, and $\theta$ is a predefined threshold value.

\subsection{The Competitive Linking Algorithm}

One major challenge of the transitive translation model is the propagation of translation errors. That is, incorrect $m$ will significantly reduce the accuracy of the translation of $s$ into $t$. A typical case is the indirect association problem (Melamed, 2000), as shown in Figure 2 in which we want to translate the term $s_{l}\left(s=s_{l}\right)$. Assume that $t_{l}$ is $s_{l}$ 's corresponding translation, but appears infrequently with $s_{l}$. An indirect association error might arise when $t_{2}$, the translation of $s_{1}$ 's highly relevant term $s_{2}$, co-occurs often with $s_{1}$. This problem is very important for the situation in which translation is a many-to-many mapping. To reduce such errors and enhance the reliability of the estimation, a competitive linking algorithm, which is extended from Melamed's work
(Melamed, 2000), is developed to determine the most probable translations.

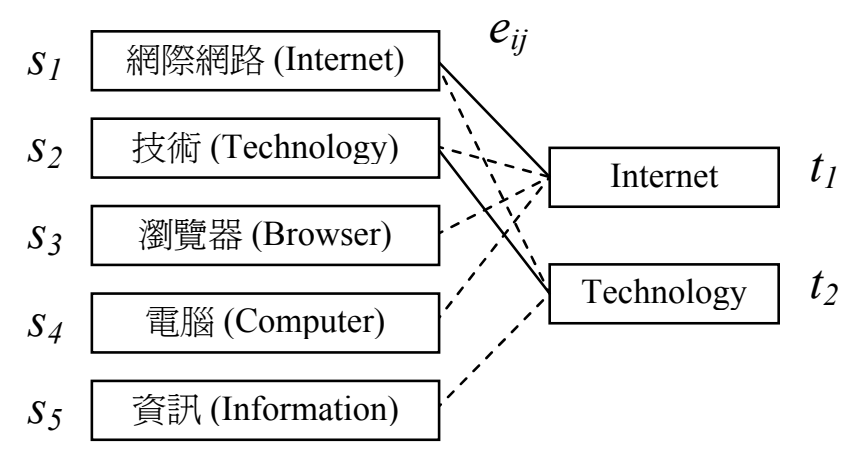

Figure 2: An illustration of a bipartite graph.

The idea of the algorithm is described below. For each translated term $t_{j} \in T$ in $l_{t}$, we translate it back into original language $l_{s}$ and then model the translation mappings as a bipartite graph, as shown in Figure 2 , where the vertices on one side correspond to the terms $\left\{s_{i}\right\}$ or $\left\{t_{j}\right\}$ in one language. An edge $e_{i j}$ indicates the corresponding two terms $s_{i}$ and $t_{j}$ might be the translations of each other, and is weighted by the sum of $S_{\text {direct }}\left(s_{i}, t_{j}\right)$ and $S_{\text {direct }}\left(t_{j}, s_{i}\right)$. Based on the weighted values, we can examine if each translated term $t_{j} \in T$ in $l_{t}$ can be correctly translated into the original term $s_{1}$. If term $t_{j}$ has any translations better than term $s_{l}$ in $l_{s}$, term $t_{j}$ might be a so-called indirect association error and should be eliminated from $T$. In the above example, if the weight of $e_{22}$ is larger than that of $e_{12}$, the term "Technology" will be not considered as the translation of “網際網路” (Internet). Finally, for all translated terms $\left\{t_{j}\right\} \subseteq T$ that are not eliminated, we re-rank them by the weights of the edges $\left\{e_{i j}\right\}$ and the top $k$ ones are then taken as the translations. More detailed description of the algorithm could be referred to Lu et al. (2004).

\section{Direct Translation}

In this section, we will describe the details of the direct translation process, i.e. the way to compute $S_{d i-}$ ${ }_{\text {rect }}(s, t)$. Three methods will be presented to estimate the similarity between a source term and each of its translation candidates. Moreover, because the searchresult pages of the term might contain snippets that do not actually be written in the target language, we will introduce a filtering method to eliminate the translation variations not of interest.

\subsection{Translation Extraction}

The Chi-square Method: A number of statistical measures have been proposed for estimating term association based on co-occurrence analysis, including mutual information, DICE coefficient, chi-square test, and log-likelihood ratio (Rapp, 1999). Chisquare test $\left(\chi^{2}\right)$ is adopted in our study because the required parameters for it can be obtained by submit- 
ting Boolean queries to search engines and utilizing the returned page counts (number of pages). Given a term $s$ and a translation candidate $t$, suppose the total number of Web pages is $N$; the number of pages containing both $s$ and $t, n(s, t)$, is $a$; the number of pages containing $s$ but not $t, n(s, \neg t)$, is $b$; the number of pages containing $t$ but not $s, n(\neg s, t)$, is $c$; and the number of pages containing neither $s$ nor $t, n(\neg s, \neg t)$, is $d$. (Although $d$ is not provided by search engines, it can be computed by $d=N-a-b-c$.) Assume $s$ and $t$ are independent. Then, the expected frequency of $(s, t)$, $E(s, t)$, is $(a+c)(a+b) / N$; the expected frequency of $(s, \neg t), E(s, \neg t)$, is $(b+d)(a+b) / N$; the expected frequency of $(\neg s, t), E(\neg s, t)$, is $(a+c)(c+d) / N$; and the expected frequency of $(\neg s, \neg t), E(\neg s, \neg t)$, is $(b+d)(c+d) / N$.

Hence, the conventional chi-square test can be computed as:

$$
\begin{aligned}
& S_{\text {direct }}^{\chi^{2}}(s, t) \\
& =\sum_{\forall X \in\{s, \neg s\}, \forall Y \in\{t, \neg t\}} \frac{[n(X, Y)-E(X, Y)]^{2}}{E(X, Y)} \\
& =\frac{N \times(a \times d-b \times c)^{2}}{(a+b) \times(a+c) \times(b+d) \times(c+d)} .
\end{aligned}
$$

Although the chi-square method is simple to compute, it is more applicable to high-frequency terms than low-frequency terms since the former are more likely to appear with their candidates. Moreover, certain candidates that frequently co-occur with term $s$ may not imply that they are appropriate translations. Thus, another method is presented.

The Context-Vector Method: The basic idea of this method is that the term $s$ 's translation equivalents may share common contextual terms with $s$ in the search-result pages, similar to Rapp (1999). For both $s$ and its candidates $C$, we take their contextual terms constituting the search-result pages as their features. The similarity between $s$ and each candidate in $C$ will be computed based on their feature vectors in the vector-space model.

Herein, we adopt the conventionaltf-idf weighting scheme to estimate the significance of features and define it as:

$$
w_{t_{i}}=\frac{f\left(t_{i}, p\right)}{\max { }_{j} f\left(t_{j}, p\right)} \times \log \left(\frac{N}{n}\right),
$$

where $f\left(t_{i}, p\right)$ is the frequency of term $t_{i}$ in search-result page $p, N$ is the total number of Web pages, and $n$ is the number of the pages containing $t_{i}$. Finally, the similarity between term $s$ and its translation candidate $t$ can be estimated with the cosine measure, i.e. $S_{\text {direct }}^{C V}(s, t)=\cos \left(c v_{s}, c v_{t}\right)$, where $c v_{s}$ and $c v_{t}$ are the context vectors of $s$ and $t$, respectively.

In the context-vector method, a low-frequency term still has a chance of extracting correct translations, if it shares common contexts with its translations in the search-result pages. Although the method provides an effective way to overcome the chi-square method's problem, its performance depends heavily on the quality of the retrieved search-result pages, such as the sizes and amounts of snippets. Also, feature selection needs to be carefully handled in some cases.

The Combined Method: The context-vector and chisquare methods are basically complementary. Intuitively, a more complete solution is to integrate the two methods. Considering the various ranges of similarity values between the two methods, we compute the similarity between term $s$ and its translation candidate $t$ by the weighted sum of $1 / R_{\chi^{2}}(s, t)$ and $1 / R_{C V}(s, t) . R_{\chi^{2}}(s, t)$ (or $R_{C V}(s, t)$ ) represents the similarity ranking of each translation candidate $t$ with respect to $s$ and is assigned to be from 1 to $k$ (number of output) in decreasing order of similarity measure $S^{x^{2}}$ direct $(s, t)$ (or $S^{c V}$ direct $(s, t)$ ). That is, if the similarity rankings of $t$ are high in both of the context-vector and chi-square methods, it will be also ranked high in the combined method.

\subsection{Translation Filtering}

The direct translation process assumes that the retrieved search-result pages of a term exactly contain snippets from a certain region (e.g. Hong Kong) and written in the target language (e.g. traditional Chinese). However, the assumption might not be reliable because the location (e.g. URL) of a Web page may not imply that it is written by the principle language used in that region. Also, we cannot identify the language of a snippet simply using its character encoding scheme, because different regions may use the same character encoding schemes (e.g. Taiwan and Hong Kong mainly use the same traditional Chinese encoding scheme).

From previous work (Tsou et al., 2004) we know that word entropies significantly reflect language differences in Hong Kong, Taiwan and China. Herein, we propose another method for dealing with the above problem. Since our goal is trying to eliminate the translation candidates $\left\{t_{j}\right\}$ that are not from the snippets in language $l_{t}$, for each candidate $t_{j}$ we merge all of the snippets that contain $t_{j}$ into a document and then identify the corresponding language of $t_{j}$ based on the document. We train a uni-gram language model for each language of concern and perform language identification based on a discrimination function, which locates maximum character or word entropy and is defined as:

$$
\operatorname{lang}\left(t_{j}\right)=\underset{l \in L}{\arg \max }\left\{\sum_{w \in N(t j)} p(w \mid l) \ln p(w \mid l)\right\},
$$

where $N\left(t_{j}\right)$ is the collection of the snippets containing $t_{j}$ and $L$ is a set of languages to be identified. The candidate $t_{j}$ will be eliminated if $\operatorname{lang}\left(t_{j}\right) \neq l_{t}$. 
To examine the feasibility of the proposed method in identifying Chinese in Taiwan, mainland China and Hong Kong, we conducted a preliminary experiment. To avoid the data sparseness of using a tri-gram language model, we simply use the above unigram model to perform language identification. Even so, the experimental result has shown that very high identification accuracy can be achieved. Some Web portals contain different versions for specific regions such as Yahoo! Taiwan (http://tw.yahoo. com) and Yahoo! Hong Kong (http://hk.yahoo.com). This allows us to collect regional training data for constructing language models. In the task of translating English terms into traditional Chinese in Taiwan, the extracted candidates for "laser" contained “雷 射” (translation of laser mainly used in Taiwan) and “激光” (translation of laser mainly used in mainland China). Based on the merged snippets, we found that “激光” had higher entropy value for the language model of mainland China while “雷射” had higher entropy value for the language models of Taiwan and Hong Kong.

\section{Performance Evaluation}

We conducted extensive experiments to examine the performance of the proposed approach. We obtained the search-result pages of a term by submitting it to the real-world search engines, including Google and Openfind (http://www.openfind.com.tw). Only the first 100 snippets received were used as the corpus.

Performance Metric: The average top- $n$ inclusion rate was adopted as a metric on the extraction of translation equivalents. For a set of terms to be translated, its top- $n$ inclusion rate was defined as the percentage of the terms whose translations could be found in the first $n$ extracted translations. The experiments were categorized into direct translation and transitive translation.

\subsection{Direct Translation}

Data set: We collected English terms from two realworld Chinese search engine logs in Taiwan, i.e. Dreamer (http://www.dreamer.com.tw) and GAIS (http://gais.cs.ccu.edu.tw). These English terms were potential ones in the Chinese logs that needed correct translations. The Dreamer log contained 228,566 unique query terms from a period of over 3 months in 1998, while the GAIS $\log$ contained 114,182 unique query terms from a period of two weeks in 1999. The collection contained a set of 430 frequent English terms, which were obtained from the 1,230 English terms out of the most popular 9,709 ones (with frequencies above 10 in both $\operatorname{logs})$. About 36\% (156/430) of the collection could be found in the LDC (Linguistic Data Consortium, http://www.ldc.upenn. edu/Projects/Chinese) English-to-Chinese lexicon with $120 \mathrm{~K}$ entries, while about $64 \%$ (274/430) were not covered by the lexicon.

English-to-Chinese Translation: In this experiment, we tried to directly translate the collected 430 English terms into traditional Chinese. Table 1 shows the results in terms of the top 1-5 inclusion rates for the translation of the collected English terms. " $\chi$ ", " $\mathrm{CV}$, and " $\chi{ }^{2}+C V$ " represent the methods based on the chisquare, context-vector, and chi-square plus contextvector methods, respectively. Although either the chi-square or context-vector method was effective, the method based on both of them $\left(\chi^{2}+\mathrm{CV}\right)$ achieved the best performance in maximizing the inclusion rates in every case because they looked complementary. The proposed approach was found to be effective in finding translations of proper names, e.g. personal names “Jordan” (喬丹, 喬登), “Keanu Reeves” (基努李維, 基諾李維), companies' names “TOYOTA” (豐田), “EPSON” (愛普生), and technical terms “EDI" (電子資料交換), “Ethernet” (乙 太網路), etc.

English-to-Chinese Translation for Mainland China, Taiwan and Hong Kong: Chinese can be classified into simplified Chinese (SC) and traditional Chinese (TC) based on its writing form or character encoding scheme. SC is mainly used in mainland China while TC is mainly used in Taiwan and Hong Kong (HK). In this experiment, we further investigated the effectiveness of the proposed approach in English-to-Chinese translation for the three different regions. The collected 430 English terms were classified into five types: people, organization, place, computer and network, and others.

Tables 2 and 3 show the statistical results and some examples, respectively. In Table 3 , the number stands for a translated term's ranking. The underlined terms were correct translations and the others were relevant translations. These translations might benefit the CLIR tasks, whose performance could be referred to our earlier work which emphasized on translating unknown queries (Cheng et al., 2004). The results in Table 2 show that the translations for mainland China and HK were not reliable enough in the top-1, compared with the translations for Taiwan. One possible reason was that the test terms were collected from Taiwan's search engine logs. Most of them were popular in Taiwan but not in the others. Only 100 snippets retrieved might not balance or be sufficient for translation extraction. However, the inclusion rates for the three regions were close in the top-5. Observing the five types, we could find that type place containing the names of well-known countries and cities achieved the best performance in maximizing the inclusion rates in every case and almost had no regional variations $(9 \%, 1 / 11)$ except 
Table 1: Inclusion rates for Web query terms using various similarity measurements

\begin{tabular}{|c|c|c|c|c|c|c|c|c|c|}
\hline \multirow{2}{*}{ Method } & \multicolumn{3}{|c|}{ Dic } & \multicolumn{3}{c|}{ OOV } & \multicolumn{3}{c|}{ All } \\
\cline { 2 - 10 } & Top-1 & Top-3 & Top-5 & Top-1 & Top-3 & Top-5 & Top-1 & Top-3 & Top-5 \\
\hline \hline$\chi^{2}$ & $42.1 \%$ & $57.9 \%$ & $62.1 \%$ & $40.2 \%$ & $53.8 \%$ & $56.2 \%$ & $41.4 \%$ & $56.3 \%$ & $59.8 \%$ \\
\hline CV & $51.7 \%$ & $59.8 \%$ & $62.5 \%$ & $45.0 \%$ & $55.6 \%$ & $57.4 \%$ & $49.1 \%$ & $58.1 \%$ & $60.5 \%$ \\
\hline$\chi^{2}+$ CV & $52.5 \%$ & $60.4 \%$ & $63.1 \%$ & $46.1 \%$ & $56.2 \%$ & $58.0 \%$ & $50.7 \%$ & $58.8 \%$ & $61.4 \%$ \\
\hline
\end{tabular}

\begin{tabular}{|c|c|c|c|c|c|c|c|c|c|}
\hline \multirow{3}{*}{ Type } & \multicolumn{9}{|c|}{ Extracted Translations } \\
\hline & \multicolumn{3}{|c|}{ Taiwan (Big5) } & \multicolumn{3}{|c|}{ Mainland China (GB) } & \multicolumn{3}{|c|}{ Hong Kong (Big5) } \\
\hline & Top-1 & Top-3 & Top-5 & Top-1 & Top-3 & Top-5 & Top-1 & Top-3 & Top-5 \\
\hline People (14) & $57.1 \%$ & $64.3 \%$ & $64.3 \%$ & $35.7 \%$ & $57.1 \%$ & $64.3 \%$ & $21.4 \%$ & $57.1 \%$ & $57.1 \%$ \\
\hline Organization (147) & $44.9 \%$ & $55.1 \%$ & $56.5 \%$ & $47.6 \%$ & $58.5 \%$ & $62.6 \%$ & $37.4 \%$ & $46.3 \%$ & $53.1 \%$ \\
\hline Place (11) & $90.9 \%$ & $90.9 \%$ & $90.9 \%$ & $63.6 \%$ & $100.0 \%$ & $100.0 \%$ & $81.8 \%$ & $81.8 \%$ & $81.8 \%$ \\
\hline Computer \& Network (115) & $55.8 \%$ & $59.3 \%$ & $63.7 \%$ & $32.7 \%$ & $59.3 \%$ & $64.6 \%$ & $42.5 \%$ & $65.5 \%$ & $68.1 \%$ \\
\hline Others (143) & $49.0 \%$ & $58.7 \%$ & $62.2 \%$ & $30.8 \%$ & $49.7 \%$ & $58.7 \%$ & $28.7 \%$ & $50.3 \%$ & $60.8 \%$ \\
\hline Total (430) & $50.7 \%$ & $58.8 \%$ & $61.4 \%$ & $38.1 \%$ & $56.7 \%$ & $62.8 \%$ & $36.5 \%$ & $54.0 \%$ & $60.5 \%$ \\
\hline
\end{tabular}

Table 3: Examples of extracted correct/relevant translations of English terms in three Chinese regions

\begin{tabular}{|c|c|c|c|}
\hline \multirow[t]{2}{*}{ English Terms } & \multicolumn{3}{|c|}{ Extracted Correct or Relevant Target Translations } \\
\hline & Taiwan (Traditional Chinese) & Mainland China (Simplified Chinese) & Hong Kong (Traditional Chinese) \\
\hline Police & 警察 (1) 警察隊 (2) 警察局 (4) & 警察 (1) 警务 (2) 公安 (4) & 警務處 (1) 警察 (3) 警司 (5) \\
\hline Taxi & 計程車 (1) 交通 (3) & 出租车 (1) 的士 (4) & 的士 (1) 的士司機 (2) 收費表 (15) \\
\hline Laser & 雷射 (1) 雷射光源 (3) 測距槍 (4) & 激光 (1) 中国 (2) 激光器 (3) 雷射 (4) & 激光 (1) 雷射 (2) 激光的 (3) 鐳射 (4) \\
\hline Hacker & 䮄客 (1) 網路 (2) 軟體 (7) & 黑客 (1) 网络安全 (5) 防火墙 (6) & 䮄客 (1) 黑客 (2) 互聯網 (9) \\
\hline Database & 資料庫 (1) 中文資料庫 (3) & 数据库 (1) 数据库维护 (9) & 資料庫 (1) 數據庫 (3) 資料 (5) \\
\hline Information & 資訊 (1) 新聞 (3) 資訊網 (4) & 信息 (1) 信息网 (3) 资讯 (7) & 資料 (1) 資訊 (6) \\
\hline Internet café & 網路咖啡 (3) 網路 (4) 網咖 (5) & 网络咖啡 (1) 网络咖啡屋 (2) 网吧 (6) & 網吧 (1) 香港 (3) 網站 (4) \\
\hline Search Engine & 搜尋器 (2) 搜尋引擎 (5) & 搜索引擎工厂 (1) 搜索引擎 (3) & 搜索器 (1) 搜尋器 $(8)$ \\
\hline Digital Camera & 相機 (1) 數位相機 (2) & 数码相机 (1) 数码影像 (6) & 像素 (1) 數碼相機 (2) 相機 (3) \\
\hline
\end{tabular}

Table 4: Inclusion rates of transitive translations of proper names and technical terms

\begin{tabular}{|c|l|l|l|l|c|c|}
\hline \multirow{2}{*}{ Type } & \multicolumn{1}{|c|}{$\begin{array}{c}\text { Source } \\
\text { Language }\end{array}$} & $\begin{array}{c}\text { Target } \\
\text { Language }\end{array}$ & $\begin{array}{c}\text { Intermediate } \\
\text { Language }\end{array}$ & Top-1 & Top-3 & Top5 \\
\hline \hline \multirow{5}{*}{ Scientist Name } & Chinese & English & None & $70.0 \%$ & $84.0 \%$ & $86.0 \%$ \\
\cline { 2 - 7 } & English & Japanese & None & $32.0 \%$ & $56.0 \%$ & $64.0 \%$ \\
\cline { 2 - 7 } & English & Korean & None & $34.0 \%$ & $58.0 \%$ & $68.0 \%$ \\
\cline { 2 - 7 } & Chinese & Japanese & English & $26.0 \%$ & $40.0 \%$ & $48.0 \%$ \\
\cline { 2 - 7 } & Chinese & Korean & English & $30.0 \%$ & $42.0 \%$ & $50.0 \%$ \\
\hline \multirow{5}{*}{ Disease Name } & Chinese & English & None & $50.0 \%$ & $74.0 \%$ & $74.0 \%$ \\
\cline { 2 - 7 } & English & Japanese & None & $38.0 \%$ & $48.0 \%$ & $62.0 \%$ \\
\cline { 2 - 7 } & English & Korean & None & $30.0 \%$ & $50.0 \%$ & $58.0 \%$ \\
\cline { 2 - 7 } & Chinese & Japanese & English & $32.0 \%$ & $44.0 \%$ & $50.0 \%$ \\
\cline { 2 - 7 } & Chinese & Korean & English & $24.0 \%$ & $38.0 \%$ & $44.0 \%$ \\
\hline
\end{tabular}

that the city “Sydney" was translated into 悉尼 (Sydney) in SC for mainland China and HK and 雪梨 (Sydney) in TC for Taiwan. Type computer and network containing technical terms had the most regional variations $(41 \%, 47 / 115)$ and type people had $36 \%(5 / 14)$. In general, the translations in the two types were adapted to the use in different regions. On the other hand, $10 \%(15 / 147)$ and $8 \%(12 / 143)$ of the translations in types organization and others, respectively, had regional variations, because most of the terms in type others were general terms such as "bank" and "movies" and in type organization many local companies in Taiwan had no translation variations in mainland China and HK.
Moreover, many translations in the types of people, organization, and computer and network were quite different in Taiwan and mainland China such as the personal name "Bred Pitt" was translated into “毕彼特” in SC and “布萊德彼特” in TC, the company name “Ericsson” into “爱立信” in SC and “易 利信” in TC, and the computer-related term “EDI" into “電子數據聯通” in SC and “電子資料交換” in TC. In general, the translations in HK had a higher chance to cover both of the translations in mainland China and Taiwan.

\subsection{Multilingual \& Transitive Translation}


Data set: Since technical terms had the most region variations among the five types as mentioned in the previous subsection, we collected two other data sets for examining the performance of the proposed approach in multilingual and transitive translation. The data sets contained 50 scientists' names and 50 disease names in English, which were randomly selected from 256 scientists (Science/People) and 664 diseases (Health/Diseases) in the Yahoo! Directory (http://www.yahoo.com), respectively.

English-to-Japanese/Korean Translation: In this experiment, the collected scientists' and disease names in English were translated into Japanese and Korean to examine if the proposed approach could be applicable to other Asian languages. As the result in Table 4 shows, for the English-to-Japanese translation, the top-1, top-3, and top-5 inclusion rates were $35 \%, 52 \%$, and $63 \%$, respectively; for the English-to-Korean translation, the top-1, top-3, and top5 inclusion rates were $32 \%, 54 \%$, and $63 \%$, respectively, on average.

Chinese-to-Japanese/Korean Translation via English: To further investigate if the proposed transitive approach can be applicable to other language pairs that are not frequently mixed in documents such as Chinese and Japanese (or Korean), we did transitive translation via English. In this experiment, we first manually translated the collected data sets in English into traditional Chinese and then did the Chinese-to-Japanese/Korean translation via the third language English.

The results in Table 4 show that the propagation of translation errors reduced the translation accuracy. For example, the inclusion rates of the Chinese-toJapanese translation were lower than those of the English-to-Japanese translation since only $70 \%-86 \%$ inclusion rates were reached in the Chinese-toEnglish translation in the top 1-5. Although transitive translation might produce more noisy translations, it still produced acceptable translation candidates for human verification. In Table 4, 45\%$50 \%$ of the extracted top 5 Japanese or Korean terms might have correct translations.

\section{Conclusion}

It is important that the translation of a term can be automatically adapted to its usage in different dialectal regions. We have proposed a Web-based translation approach that takes into account limited bilingual search-result pages from real search engines as comparable corpora. The experimental results have shown the feasibility of the automatic approach in generation of effective translation equivalents of various terms and construction of multilingual translation lexicons that reflect regional translation variations.

\section{References}

L. Borin. 2000. You'll take the high road and I'll take the low road: using a third language to improve bilingual word alignment. In Proc. of COLING-2000, pp. 97-103.

P. F. Brown, J. Cocke, S. A. D. Pietra, V. J. D. Pietra, F. Jelinek, J. D. Lafferty, R. L. Mercer, and P. S. Roossin. 1990. A statistical approach to machine translation. Computational Linguistics, 16(2):79-85.

Y.-B. Cao and H. Li. 2002. Base noun phrase translation using Web data the EM algorithm. In Proc. of COLING-2002, pp. 127-133.

P.-J. Cheng, J.-W. Teng, R.-C. Chen, J.-H. Wang, W.-H. Lu, and L.-F. Chien. 2004. Translating unknown queries with Web corpora for cross-language information retrieval. In Proc. of ACM SIGIR-2004.

P. Fung and L. Y. Yee. 1998. An IR approach for translating new words from nonparallel, comparable texts. In Proc. of ACL-98, pp. 414-420.

T. Gollins and M. Sanderson. 2001. Improving cross language information with triangulated translation. In Proc. of ACM SIGIR-2001, pp. 90-95.

J. Halpern. 2000. Lexicon-based orthographic disambiguation in CJK intelligent information retrieval. In Proc. of Workshop on Asian Language Resources and International Standardization.

A. Kilgarriff and G. Grefenstette. 2003. Introduction to the special issue on the web as corpus. Computational Linguistics 29(3): 333-348.

J. M. Kupiec. 1993. An algorithm for finding noun phrase correspondences in bilingual corpora. In Proc. of $A C L$ 93, pp. 17-22.

W.-H. Lu, L.-F. Chien, and H.-J. Lee. 2004. Anchor text mining for translation of web queries: a transitive translation Approach. ACM TOIS 22(2): 242-269.

W.-H. Lu, L.-F. Chien, and H.-J. Lee. 2002. Translation of Web queries using anchor text mining. ACM TALIP: 159-172.

I. D. Melamed. 2000. Models of translational equivalence among words. Computational Linguistics, 26(2): 221249.

J.-Y. Nie, P. Isabelle, M. Simard, and R. Durand. 1999. Cross-language information retrieval based on parallel texts and automatic mining of parallel texts from the Web. In Proc. of ACM SIGIR-99, pp. 74-81.

R. Rapp. 1999. Automatic identification of word translations from unrelated English and German corpora, In Proc. of ACL-99, pp. 519-526.

P. Resnik. 1999. Mining the Web for bilingual text. In Proc. of ACL-99, pp. 527-534.

M. Simard. 2000. Multilingual Text Alignment. In "Parallel Text Processing”, J. Veronis, ed., pages 49-67, Kluwer Academic Publishers, Netherlands.

F. Smadja, K. McKeown, and V. Hatzivassiloglou. 1996. Translating collocations for bilingual lexicons: a statistical approach. Computational Linguistics, 22(1): 1-38.

B. K. Tsou, T. B. Y. Lai, and K. Chow. 2004. Comparing entropies within the Chinese language. In Proc. of IJCNLP-2004.

C. C. Yang and K.-W. Li. 2003. Automatic construction of English/Chinese parallel corpora. JASIST 54(8): 730-742. 